\title{
CONTROL METHODS AND ACTIVE FILTER TOPOLOGIES APPLIED FOR FLICKER MITIGATION
}

\author{
Jaroslav Dolezal", Josef Tlusty", Viktor Valouch ${ }^{* *}$ \\ "Department of Power Engineering, Faculty of Electrical Engineering, CTU Technická 2, 16627 Prague 6, Czech \\ RepublicE-mail: tlusty@fel.cvut.cz,dolezalj@fel.cvut.cz \\ ** Institute of Electrical Engineering, Academy of Sciences of the Czech republic Dolejškova 5, 18202 Praha 8, Czech \\ RepublicE-mail: valouch@iee.cas.cz
}

\begin{abstract}
A linearised model of the power system with the Electrical Arc Furnace (EAF) and the Unified Power Quality Conditioner (UPQC) has been used in the frequency domain for finding an optimum control strategy of the UPQC. The analysis indicates that the most effective may be such an application of the UPQC where the parallel Active Filter (AF) compensates the whole reactive power consumed by the EAF and delivers to the network a capacitive power to hold the voltage at the PCC constant, while the series AF compensates the fluctuating active power of the EAF. Copyright (C) 2005 IFAC
\end{abstract}

Keywords: electrical arc furnace; flicker; linearised model; unified power quality conditioner; parallel and series active filter

\section{INTRODUCTION}

A voltage fluctuation that results in flicker is caused by Electrical Arc Furnace (EAF) power fluctuation. The Unified Power Quality Conditioner (UPQC) belongs to the group of power electronics-based compensation devices called in summary Flexible AC Transmission System (FACTS). The FACTS offers many compensation functions for power transmission systems and represents the most progressive way to increase power quality in the systems. The UPQC can be an effective means to suppress voltage fluctuation connected with the function of the EAF. Hitherto, the flicker has been compensated for mainly by using Static Var Compensators (SVC), whose capability to eliminate voltage subharmonics is limited by the frequency up to $10 \mathrm{~Hz}$ at maximum. Recently, some studies and tests have been done to prove advantages of active filters (AF) over SVC in this field. The UPFC consists of the parallel and series AF, so it offers more possibilities to solve instantaneous active and reactive power fluctuation than either the parallel or series AF alone.

A model of a power system with an electric arc furnace was developed for a typical metallurgical plant.

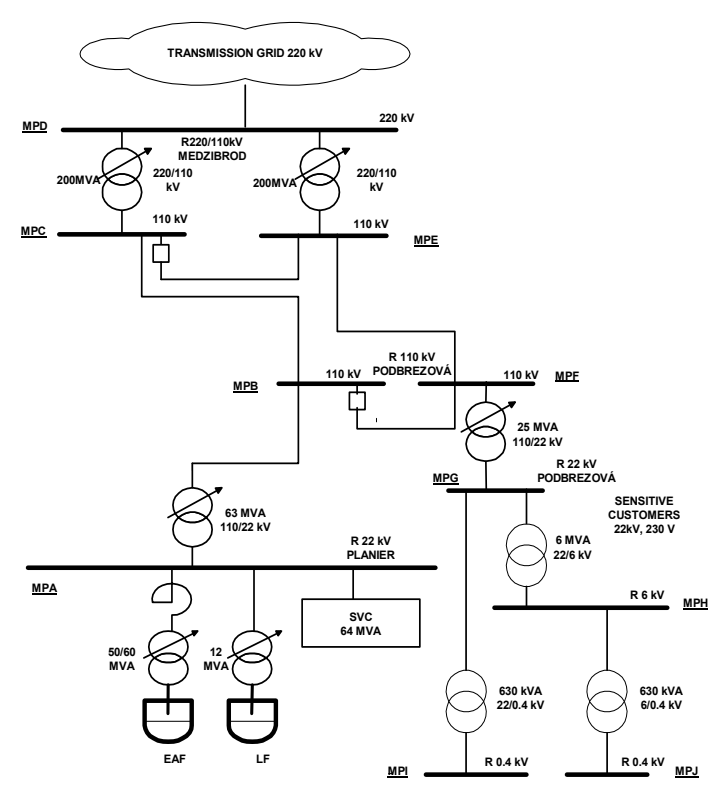

Fig. 1. Distribution system

Fig. 1 shows the location of the EAF in the part of the distribution network with low short circuit power. The EAF has been equipped with the SVC and four passive filters tuned to the $2,3,4,5^{\text {th }}$ harmonics. 
Measurement with different values of current, of short-circuit ratios at the PCC, of arc length and voltage ratios of the furnace transformer has been done to derive the arc-furnace characteristic curves, Fig. 2.

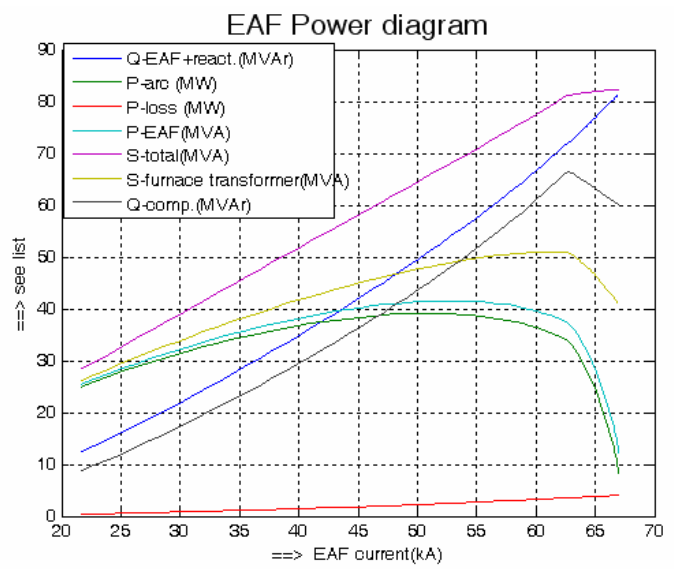

Fig. 2. Arc-furnace characteristic

The values of all parameters used for model were got from a real series of measurements of the electric arc furnace and its network. In order to illustrate the limits of regulation of flicker with the help of an SVC controlled by a classical regulator without a predictor we can show the Flicker Suppression Factor (F.S.F.), Fig. 3.

\section{F. S. F. for PID regulator of SVC}

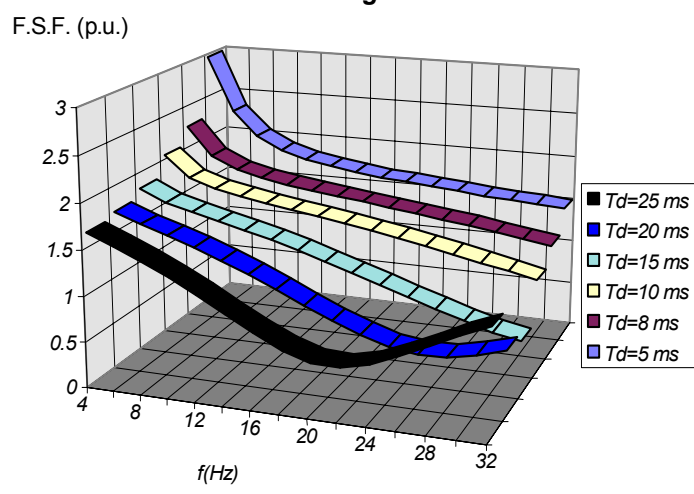

Fig.3. Flicker Suppression Factor for SVC in operation.

The results show a low Flicker Suppression Factor of the SVC for this supply system due to time delay Td in the control loop of the SVC.

The current requirements and standards have been fulfilled from the reactive power compensation, unbalance and harmonics point of view, but not for the voltage flicker compensation, especially for subharmonics above $5 \mathrm{~Hz}$. Thanks to the use of an $\mathrm{AF}$, excellent real time operation capability of voltage flicker compensation is supposed to be viable even for higher order subharmonics.

The paper deals with the analysis of topologies and control strategies of the UPQC for the flicker compensation.

\section{UPQC USED IN AC POWER SYSTEM WITH EAF}

Fig. 4 shows a possible equivalent circuit of UPQC implementation into a power system with the EAF. The series and parallel AF can be viewed as a voltage source and a current source, respectively.

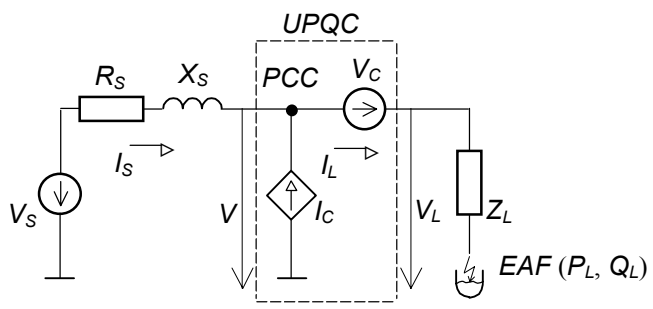

Fig.4. AC power supply of EAF with specific UPQC used.

The scheme is simplified to make a basic analysis easier. Usually, a block of traditional passive filters (PF) tuned to some harmonics is connected in parallel with the EAF.

The parameters $R_{S}, X_{S}$ represent the impedance of the mains, while $Z_{L}$ is the impedance of the transformer and cables of the EAF. The aim of the UPQC is to compensate for the voltage $V_{L}$ fluctuation to hold the voltage $V$ at the Point of Common Coupling (PCC) constant. Without using any compensation device (SVC, AF, UPQC) the current $I_{S}\left(=I_{L}\right.$ in such a case) and thus also the voltage $V$ at the PCC will fluctuate and provoke the flicker in a distribution system connected to the PCC.

In [De Préville,2001], the condition for attaining $|\boldsymbol{V}|=\left|\boldsymbol{V}_{S}\right|$, no matter how high is the current $\boldsymbol{I}_{S}$, was presented. If we put the vector $\boldsymbol{V}(=V)$ into the line of the axis $d$ of the reference frame rotating with the fundamental frequency $\omega_{1}$, the condition reads

$$
I_{S q}=\frac{R_{S}}{X_{S}} I_{S d}+\frac{V_{S}}{2 S_{C C}} I_{S d}^{2}
$$

where $V_{S}=\left|V_{S}\right|$ and $S_{C C}=V_{S}^{2} / Z_{S}$ is the short circuit power of one phase at the PCC. The condition (1) holds for steady-states and we will consider the symmetrical system in the following analysis.

The EAF has been usually represented by either an ideal voltage or current source [Bester et al, 1999], [ Fugita, and Akagi, 1998]. This approach is not adequate enough to reflect the real function of the EAF. For some simulation studies the Cassie-Mayr formula was successfully used [Larsson and Ekstrom, 1997],[ Zouti and Saadate, 1997]. Unfortunately this representation is not linear. The formula in its simplified version reads (in the static reference frame)

$$
\begin{gathered}
L_{L} \frac{d \boldsymbol{i}_{L}}{d t}=-\left(R_{L}+r\right) \boldsymbol{i}_{L}+\boldsymbol{v}_{L} \\
T_{A} \frac{d r}{d t}=r\left[1-\frac{1}{\boldsymbol{v}_{a}^{2}}\left(r \boldsymbol{i}_{L}\right)^{2}\right]
\end{gathered}
$$


where $R_{L}+\mathrm{j} \omega_{1} L_{L}=Z_{L}, T_{A}$ is the deionising time constant and $v_{A}$ the static voltage of the arc. The variable $r$ represents the resistance of the arc, which is varying in transient processes and is connected with the arc length. The current and connected voltage fluctuation of the EAF, provoked by the change of the arc length, may be represented by the variation of $\boldsymbol{v}_{A}$. By using the perturbation method, the system (2), (3) may be linearized and used in models of the power system.

Fig. 5 shows the simulated phase current of the EAF ( $R_{L}=0.005, L_{L}=0.05, R=0.99, T_{A}=2 \pi$, all in p.u.). The sinusoidal variation of the voltage $\Delta v_{A d}$ was assumed (of the magnitude of 0.1 p.u., $\omega_{k}=0.1$ p.u.), while the voltage components $\Delta v_{L d}, \Delta v_{L q}$ of the same frequency $\omega_{k}$ were zero. The magnitude $I_{L}$ of the load current of the fundamental frequency $\omega_{1}$ was set as nominal one. It is evident that the simulation of the EAF is possible even in the frequency domain by using the $\vartheta$ linearised model. By using the parameters of the EAF, the flicker phenomenon of the EAF can be represented by a proper variation $\Delta v_{A d}$ of the arc voltage.

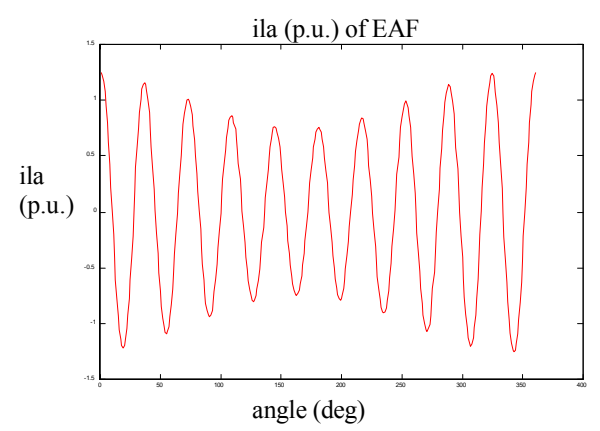

Fig.5. EAF phase current simulated by using linearised EAF model.

\section{RESULTS OF FLICKER MEASUREMENT}

Flicker measurements have been carried out in the Slovak network in order to obtain experimental data for a case study of an UPQC application.

There is a $110 \mathrm{kV}$ distribution system in Central Slovakia that is supplied from Medzibrod's $220 \mathrm{kV}$ substation by a 200MVA $220 / 110 \mathrm{kV}$ transformer. A UHP 60 MVA electrical arc furnace is installed in this area, and all the other power supply customers utilise $40 \mathrm{MW}$. The arc furnace is a dominant load and affects the power quality of all customers in the area.

The measurement was performed at the beginning of the melting process in the EAF after elapsing a few first transients when no material is even partly melt. This regime of the EAF is the most complicated and the highest amplitudes of subharmonic voltage components are just produced in that mode of operation. The measurement, whose results of the analysis are presented, was realised with the operation of the SVC, 90 MVA that involved also the series static filters tuned at the $2^{\text {nd }}, 3^{\text {rd }}, 4^{\text {th }}$ harmonics. Thus, dominant harmonics were suppressed as well, which made the next analysis more feasible.

Fig. 6 presents the results of the analysis of measurement on the EAF, 60 MVA in the steelworks with the SVC and without any AF. The amplitudes of subharmonic voltages of the positive- and negative-sequence are shown. The flicker represents the amplitude modulation of the fundamental frequency

voltage component, which results in the existence of the fundamental voltage component with a symmetrical spectrum in the network. That is why resulting voltage components with frequencies near $50 \mathrm{~Hz}$ are connected with the flicker of the lowest frequencies above $0 \mathrm{~Hz}$.
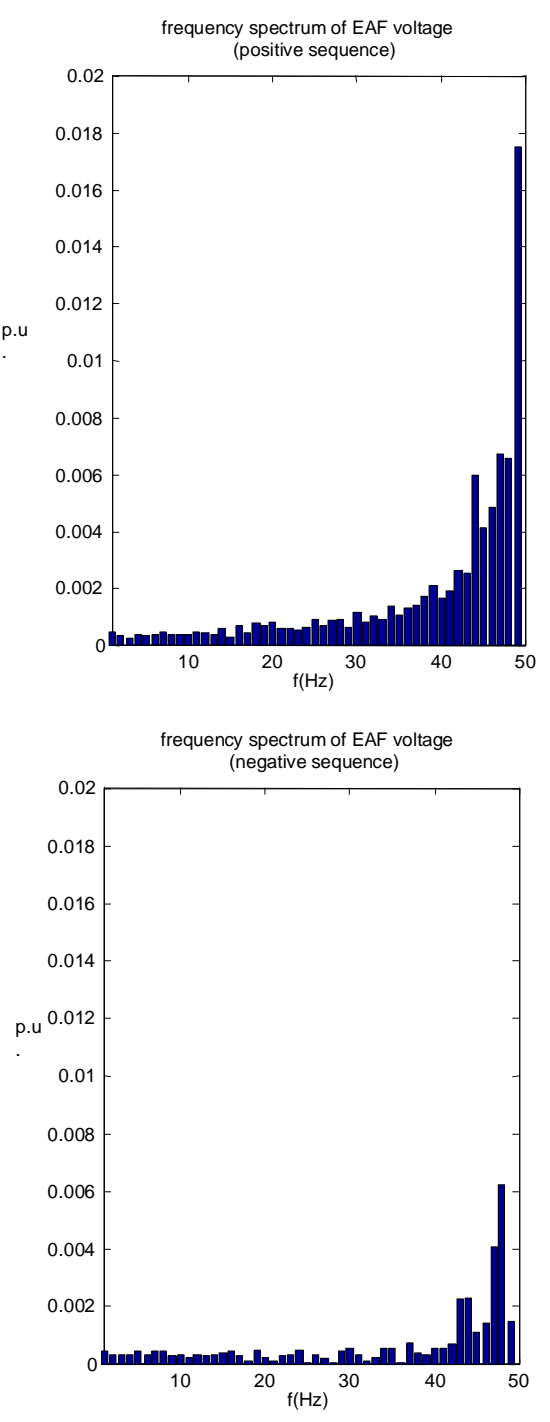

a)

b)

Fig.6. Measured frequency spectra of the EAF voltage (positive and negative sequence) 


\section{CONTROL SYSTEM OF UPQC}

Based on the analysis and equations presented a linearised model of the system in Fig. 4 may be developed in the frequency domain. Except the equations (1),(3), other equations describing the power circuit in Fig. 4 and the control system in Fig. 7 (including specific signal filters) are linear so they hold their forms regardless the absolute or perturbed signals are in question. The model makes it possible to analyse basic properties of the system for perturbed signals and different control topologies and parameters.
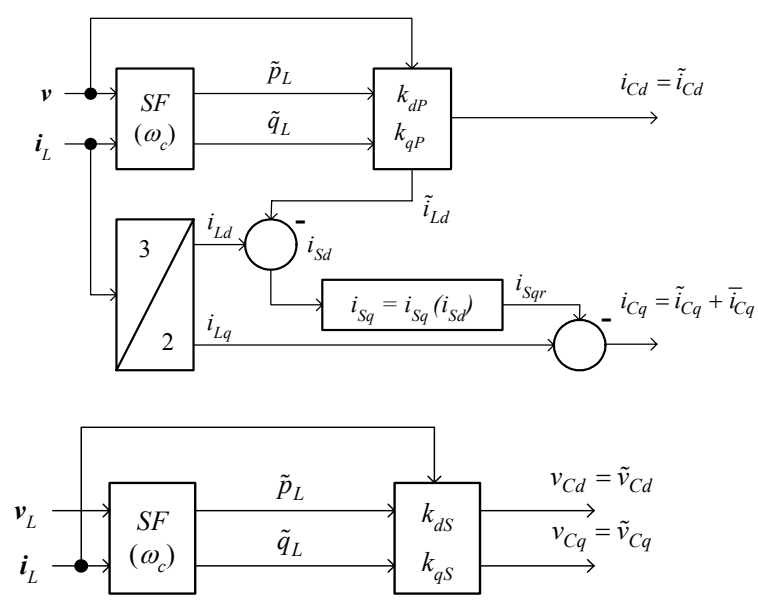

b)

Fig.7. Block diagram of the control system of a) parallel part of UPQC, b) series part of UPQC

Fig. 7 shows block diagrams of the control systems of the parallel (a) and series part (b) of the UPQC. The compensation of fluctuating (subharmonic) components of active and reactive powers of the EAF uses the concept of the Instantaneous Reactive Power (IRP) theory [Akagi, 1996]. The fluctuating power components may be obtained by using appropriate Signal Filters (SF) with a cut-off frequency $\omega_{c}$. The coefficients $k_{d p}, k_{q p}, k_{d S}, k_{q S}$ determine amount of respective fluctuating power components that should be compensated for by the parallel and series part of the UPQC. The control strategy of the parallel part of the UPQC involves also the algorithm for attaining $|\boldsymbol{V}|=\left|\boldsymbol{V}_{S}\right|$ in steady states, which has been described before. The variable $i_{S q r}$ represents the reference $q$-component of the current $\boldsymbol{i}_{S}$ of the supply system, demanded for fulfilling the previous condition (1) for basic voltage harmonics.

The system in Fig. 4 with the control strategy presented by the block diagrams in Fig. 7 may be represented, assuming that all four signal filters are of the first order, by the system of 9 state equations and 4 algebraic equations.
By substituting $\frac{\mathrm{d}}{\mathrm{d} t} \rightarrow \mathrm{j} \omega_{k}$ we can obtain the system of complex algebraic equations that describes the behaviour of the system (Fig. 4) with the controller (see Fig. 7) in the frequency domain for the frequency $\omega_{k}$ of the flicker.

\section{SIMULATION RESULTS}

Fig. 8 shows how the EAF voltage fluctuation $\Delta v_{A d}$ propagates and results in a voltage fluctuation at the PCC. The sinusoidal variation of the voltage $\Delta v_{A d}$ was assumed (of the magnitude of 0.05 p.u.).

The frequency in Fig. 8 means the frequency of the flicker that is the frequency of the amplitude modulation. No part of the UPQC is supposed to be in the function. It is evident that even for the constant amplitude of the arc voltage $\Delta v_{A d}$ the developed linearised EAF model can represent EAF behaviour quite well (compare Figs.6,8). For exact representation of the EAF the amplitude of the voltage $\Delta v_{A d}$ may be adjusted in accordance with measured EAF spectra.

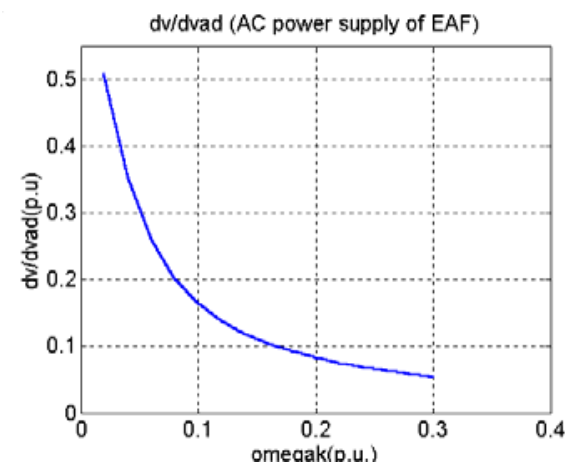

Fig.8. Propagation of amplitude of the voltage $\Delta v_{A d}$ fluctuation to the PCC versus frequency of the flicker .

Fig. 9 shows the apparent power of the UPQC demanded for complete compensation of the voltage fluctuation at the PCC. The rated power of the UPQC belongs among main criteria showing how effective the compensation strategy is and determines actually the feasibility of a project. Again, the linearised model of the system with the same parameters was used $\left(R_{L}=0.005, L_{L}=0.05, R\right.$ $=0.99, T_{A}=2 \pi, R_{S}=0.005, L_{S}=0.05, V_{\mathrm{S}}=1, P_{\mathrm{L}}=$ 1 , power factor $=0.95, \Delta v_{A d}=0.05$, all in p.u.), supposing that the EAF works at its rated mode of operation. An ideal detection of fluctuating power components of the EAF is assumed.

First, only the parallel part of the UPQC is in function $\left(I_{S q}=I_{S q}\left(I_{S d}\right), k_{d P}=1, k_{q P}=1, k_{d S}=k_{q S}=0\right)$. Thus, the parallel AF compensates the whole 
reactive power consumed by the EAF, delivers to the network a capacitive power to hold the voltage at the PCC constant, and compensates the fluctuating active power of the EAF.

Then, both parts of the UPQC are supposed to be in the function, but the whole fluctuating active power of the EAF is compensated for by the series AF only $\left(I_{S q}=I_{S q}\left(I_{S d}\right), k_{d P}=0, k_{q P}=1, k_{d S}=1, k_{q S}=0\right)$. Comparing both the options we see how different total apparent power of the UPQC is. Naturally, the analysis considers only the flow of fluctuating components, so the rated power of the real parallel $\mathrm{AF}$ in both the cases must be high enough to compensate also the voltage drop at the PCC.

Tab. 1 compares the data obtained for the selected frequency $\omega_{k}=0.1$ p.u. if the both mentioned strategies and a special one for the series AF alone $\left(k_{d S}=1\right)$ have been used.
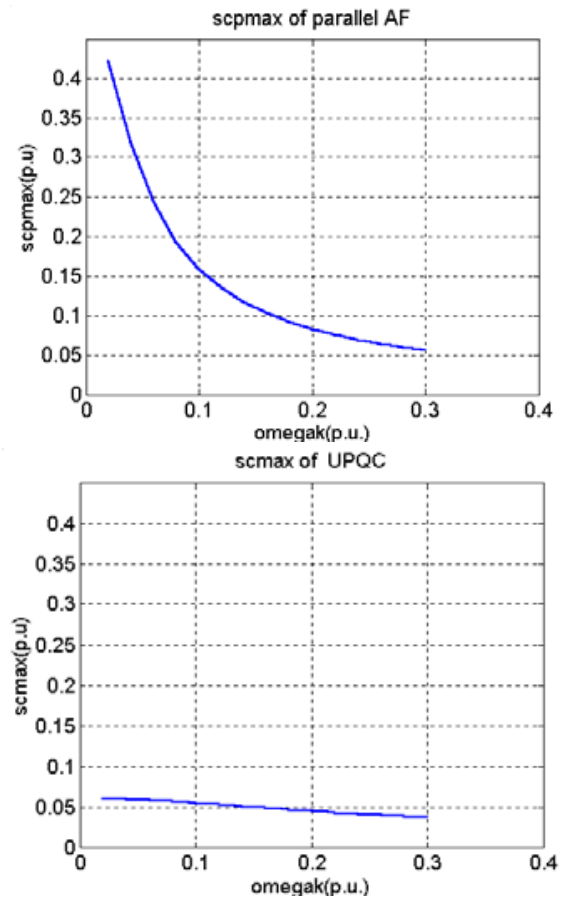

Fig. 9. Demanded power of UPQC for full compensation of the voltage subharmonic component at $\mathrm{PCC}$; a) only parallel AF in operation $\left(I_{S q}=I_{S q}\left(I_{S d}\right), k_{d P}=k_{q P}=1, k_{d S}=k_{q S}=\right.$ $0)$, b) parallel as well as series AF in operation $\left(I_{S q}=I_{S q}\left(I_{S d}\right), k_{d P}=0, k_{q P}=1, k_{d S}=1, k_{q S}=0\right)$

Table 1 Demanded powers (p.u.) of the parallel and series part of UPQC for different control strategies (without any signal filter) ensuring nearly full compensation of the voltage fluctuation

$$
\left(\Delta v_{A d}=0.05 \text { p.u., } \omega_{k}=0.1 \text { p.u. }\right)
$$

\begin{tabular}{|l|l|l|l|}
\hline Control strategy & $S_{C P}$ & $S_{C S}$ & $S_{C M A X}$ \\
\hline $\mathrm{AF}_{P}\left(I_{S q}=I_{S q}\left(I_{S d}\right), k_{d P}=\right.$ & 0.16 & & 0.16 \\
\hline $\mathrm{AF}_{S}\left(k_{d S}=1\right)$ & & 0.047 & 0.047 \\
\hline $\mathrm{AF}_{P}\left(I_{S q}=I_{S q}\left(I_{S d}\right), k_{q P}=\right.$ & 0.01 & 0.045 & 0.055 \\
\hline
\end{tabular}

Tab. 2 summarizes data obtained by the simulation of a nonlinear model based on (1)-(3). The conditions were the same as those used for Tab. I, which were obtained on the basis of the linearized model.

We can come to the conclusion that the linearized model is precise enough to predict basic results according to impacts of different control strategies of UPQC as well as influence of their parameters on the trade-off of power components flowing through the branches of the system (see Fig. 4).

Fig. 10 shows the rate $\Delta v / \Delta v_{A d}$ (a) and $S_{C M A X}$ (b) versus $\omega_{k}$ and $k_{d P}$ for the same parameters and conditions as those used above, but the signal filters of the first order with the cut-off frequency $\omega_{c}=0.02$ p.u. were used. Only the $\mathrm{AF}_{p}$ was in operation $\left(1^{\text {st }}\right.$ row in Tab. 1).

Fig. 11 presents similar plots, but now for the $\mathrm{AF}_{p}+\mathrm{AF}_{S}$ being in operation ( $3^{\text {rd }}$ row in Tab. 1).

By comparing Fig. 9, Tab. 1 with Fig. 10b), Fig. $11 b$ ), we see that the demanded powers $S_{C P}$ MAX, $S_{C S M A X}$ are not substantially influenced by the used signal filters.

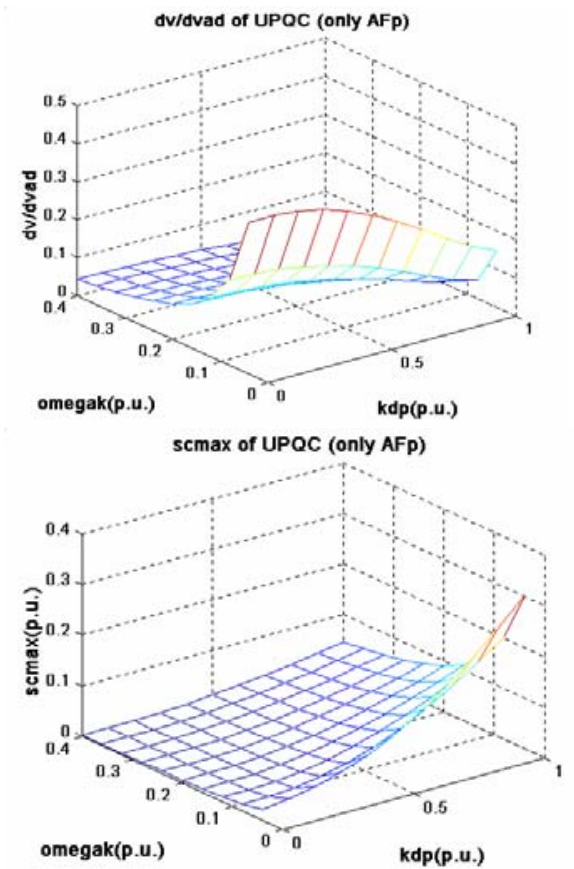

Fig. 10. $\Delta v / \Delta v_{A d}$ (a) and $S_{\mathrm{C} \operatorname{MAX}}$ (b) of UPQC (only $\mathrm{AF}_{p}$ in function) with signal filters $\left(\omega_{\mathrm{c}}=0.02\right.$ p.u.)

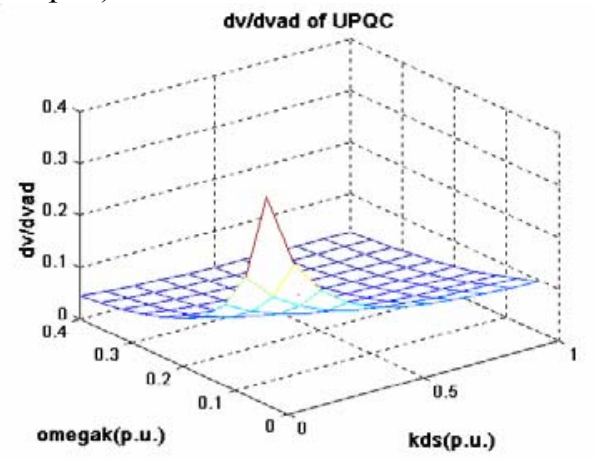




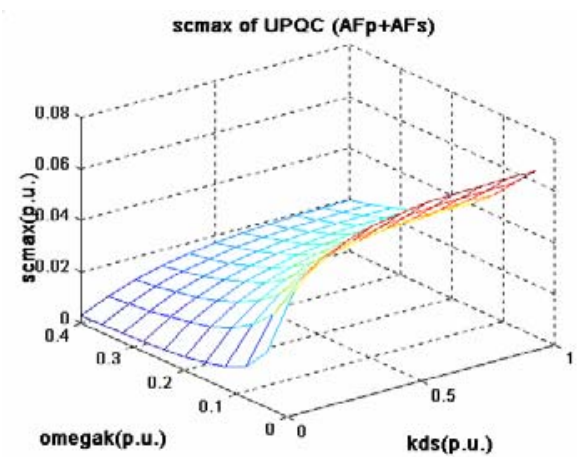

Fig. 11. $\Delta v / \Delta v_{A d}$ (a) and $S_{\mathrm{C} \text { MAX }}$ (b) of UPQC $\left(\mathrm{AF}_{p}+\right.$ $\left.\mathrm{AF}_{S}\right)$ with signal filters $\left(\omega_{\mathrm{c}}=0.02\right.$ p.u. $)$

On the other hand, Fig. 10a), 11a) show that the fluctuating component of the voltage $\Delta v$ at the PCC cannot be suppressed completely in a real system, even for $k_{d P}$ or $k_{d S}$ approaching the value 1 ( $\Delta v / \Delta v_{A d}>0$ for those values). Figs. 10, 11 also elucidate how the values of $\omega_{k}$ and $k_{d P}, k_{d S}$ influence both the key performance criteria $\Delta v / \Delta v_{A d}, S_{C M A X}$ of the UPQC with the different control strategies.

Table 2. Demanded powers (p.u.) of the parallel and series part of UPQC and amplitude (p.u.) of voltage fluctuation at PCC for different control strategies (with signal filters). Results of simulation are based on non-linear model ( $\Delta v_{A d}=0.05$ p.u., $\omega_{k}=0.1$ p.u. $)$

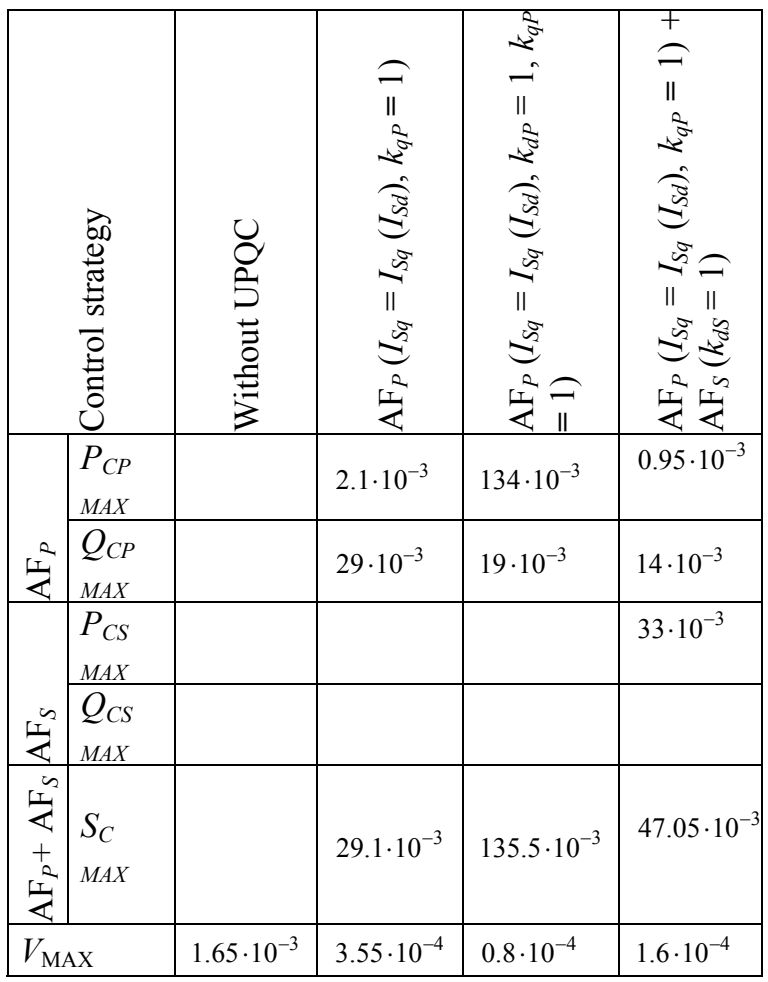

Thus, the methodology presented can make it possible to analyse the behaviour of the power and control circuitry for EAF flicker mitigation and to find an optimum control strategy and parameters.

\section{CONCLUSION}

The voltage and current components measured in the steelworks with the EAF in Central Slovakia were analysed and used as a ground for the study of the promising control strategy of the UPQC. The UPQC should improve the voltage fluctuation in the frequency range above $5 \mathrm{~Hz}$ where the current SVC has became ineffective.

The presented analysis indicates that the most effective is the compensation for both the active and reactive fluctuating EAF power components with using the strategy (1) at the same time. From the point of view of the low UPQC rated power, the better then others is the application of the UPQC where the parallel AF compensates the whole reactive power consumed by the EAF and delivers to the network a capacitive power to hold the voltage at the PCC constant, while the series AF compensates the fluctuating active power of the EAF.

\section{REFERENCES}

Akagi, H. (1996), New Trends in Active Filters for Power Conditioning, IEEE Transactions on IA32, No. 6, 1996, 1312-1322.

Bester, D. D.. Le Roux, A. D. Monston, H.T. Enslin,J. H. R.,(1999), "Evaluation of PowerRatings for Active Power Quality Compensators," EPE 1999, Lausanne, Switzerland, 1999, CD-ROM.

De Préville,G., (2001), Flicker Mitigation, "Application to a STATCOM," EPE 2001, Graz, Austria, September 2001, CD-ROM.

Fugita, H., Akagi,H.,(1998),“The Unified Power Quality conditioner. The Integration of Seriesand Shunt-Active Filters," IEEE Trans. On PE13, No.2, March 1998, 315-322.

Larsson, T., Ekstrom, A.,(1997),“A PWM-Operated Voltage Source Converter for Flicker Mitigation," EPE 1997, Trondheim, Norway, 1997, 3.1.16-3.1.20.

Zouti, M., Saadate, S.,(1997), "UPFC Application to Suppress Flicker nn Arc Furnace Supply System," EPE 1997, Trondheim, Norway, 1997, 3.983-3.987.

Acknowledgement

The financial support of the Grant Agency of the Czech Republic (project No.102/ 03/1551) is highly acknowledged. 